\title{
Downregulation of miR-195 promotes prostate cancer progression by targeting HMGA1
}

\author{
XIAOWEN ZHANG ${ }^{1,2^{*}}$, TAO TAO ${ }^{1,2^{*}}$, CHUNHUI LIU $^{1,2^{*}}$, HAN GUAN $^{1,2}$, \\ YEQING HUANG ${ }^{1,2}, \mathrm{BIN} \mathrm{XU}^{1-3}$ and MING $\mathrm{CHEN}^{1-3}$ \\ ${ }^{1}$ Department of Urology, Zhongda Hospital, School of Medicine, Southeast University, Nanjing, Jiangsu 210009; \\ ${ }^{2}$ Institute of Urology, Southeast University, Nanjing, Jiangsu 210009; ${ }^{3}$ Surgical Research Center, \\ Medical School, Southeast University, Nanjing, Jiangsu 210009, P.R. China
}

Received December 28, 2015; Accepted February 11, 2016

DOI: $10.3892 /$ or.2016.4797

\begin{abstract}
Aberrant deregulation of microRNA-195 (miR-195) is associated with tumorigenesis and the development of cancer. However, its expression and function in prostate cancer (PCa) remain to be elucidated. In the present study, we found that miR-195 expression levels were decreased in human PCa samples and were correlated with patient prognosis. miR-195 overexpression inhibited cell proliferation, cell cycle progression and tumorigenesis via directly targeting HMGA1. Downregulation of HMGA1 expression had an effect similar to miR-195 in the PCa cells. In clinical specimens, HMGA1 was overexpressed in castration-resistant prostate cancer when compared with its levels in benign prostate hyperplasia and androgen-dependent prostate cancer, and its expression levels were inversely correlated with overall survival and biochemical relapse-free survival. In summary, our study suggests that miR-195 functions as a tumor-suppressor gene by downregulating HMGA1 and can be used as a potential target in the treatment of PCa.
\end{abstract}

\section{Introduction}

Prostate cancer (PCa) is the most commonly diagnosed solid organ malignancy in the United States and remains the second leading cause of cancer-related death among American men. Approximately 29,480 deaths due to prostate cancer were recorded in the US in 2014 (1). Prostate cancer deaths are typically the result of metastatic castration-resistant prostate cancer (mCRPC), and historically the median survival for

Correspondence to: Dr Bin Xu or Dr Ming Chen, Department of Urology, Affiliated Zhongda Hospital of Southeast University, 87 Dingjia Bridge Hunan Road, Nanjing, Jiangsu 210009, P.R. China E-mail: xb15896450810@126.com

E-mail: mingchenseu@126.com

*Contributed equally

Key words: microRNA, miR-195, prostate cancer, HMGA1, cell cycle men with mCRPC is less than 2 years. Similar to other human diseases, $\mathrm{PCa}$ is associated with a wide spectrum of genetic aberrations. However, the mechanisms implicated in the initiation and progression of PCa remain unclear (2).

MicroRNAs (miRNAs) are small (18 to 24 nucleotides in length), single-stranded, endogenous non-coding RNAs that regulate gene expression post-transcriptionally. The aberrant expression of miRNAs is closely associated with the proliferation, cell cycle, apoptosis, differentiation, migration, metabolism and prognosis of various types of cancers, including PCa (3-6). miR-195 is a member of the miR-15/16 family, which consists of a group of miRNAs (miR-195, miR-15a, miR-15b, miR-16-1 and miR-16-2) that share a similar seed sequence (7). The sequence of mature miR-195 is conserved across mammalian species (8). miR-195 has been reported to be deregulated in certain types of cancer, including upregulation in chronic and acute lymphocytic leukemia (9) and metastatic melanoma (10) but is downregulated in adrenocortical, hepatocellular carcinoma and squamous cell carcinoma (11-13). However, the exact role of miR-195 in PCa remains elusive.

In this study, we found that miR-195 expression levels were decreased in human PCa samples and positively correlated with prognosis. Next, we used RNA sequence and bioinformation to screen the biological effect of miR-195 in PCa cells and found miR-195 may influence cell cycle. Further study showed that overexpression of miR-195 inhibited cell proliferation, cell cycle progression, and tumorigenesis in vitro and in vivo. In addition, HMGA1 was identified as the target gene of miR-195 in $\mathrm{PCa}$ and its high expression was inversely correlated with the prognosis of PCa patients. Downregulation of the expression of HMGA1 had an effect similar to that of miR-195 in the PCa cells. All of these results indicate that miR-195 and its downstream target gene HMGA1 can be used to predict the prognosis or even suppress the development of PCa.

\section{Materials and methods}

Data mining and bioinformation analysis. Gene expression data were downloaded from the GSE35988 (http://www. ncbi.nlm.nih.gov/geo/query/acc.cgi?acc=GSE35988) (14) and GSE21034 (http://www.ncbi.nlm.nih.gov/geo/query/acc. 
cgi?acc=GSE21034) (15). Detailed information on patients can be obtained from the Oncomine database (https://www.oncomine.org/resource/main.html). Gene set enrichment analysis (GSEA) was used to identify pathway gene sets that were correlated with the miR-195 expression profile (http://www. broadinstitute.org/gsea/index.jsp). The gene sets were derived from the Molecular Signatures Database. The normalized ES was used to compare the analysis results across the various gene sets.

Cell culture. Human prostate cancer cells, DU-145 and PC-3, were purchased from Shanghai Cell Bank, Chinese Academy of Sciences. DU-145 cells were cultured in Dulbecco's modified Eagle's medium (DMEM; Hyclone, Beijing, China) plus $10 \%$ fetal bovine serum (FBS; Hyclone, Gaithersburg, $\mathrm{MD}$, USA), $100 \mathrm{mg} / \mathrm{ml}$ streptomycin and $100 \mathrm{U} / \mathrm{ml}$ penicillin (Hyclone, Beijing, China). PC-3 cells were cultured in DMEM/F12 (Hyclone, Beijing, China) medium supplemented with $10 \% \mathrm{FBS}$ and antibiotics. Cells were incubated at $37^{\circ} \mathrm{C}$ in a humidified atmosphere of $5 \% \mathrm{CO}_{2}$ in air.

miRNA/siRNA transfections. Cells were plated in 6-well plates in their normal growth medium without antibiotics for $24 \mathrm{~h}$ before transfections. Transient transfections of miRNA mimics/siRNA (both from GenePharma) were carried out using Lipofectamine 2000 (Invitrogen, Carlsbad, CA, USA) according to the manufacturer's instructions, when cells reached $50-70 \%$ confluency. miR-195 mimics, the negative control (miR-NC), HMGA1 siRNA or the universal scrambled negative control (NC siRNA) were purchased from GenePharma (Shanghai, China).

MTT assay. The transfected cells were plated at 3,000/well in 96-well plates. Every $24 \mathrm{~h}$ after plating, respectively, $20 \mu \mathrm{l}$ of $5 \mathrm{mg} / \mathrm{ml}$ MTT in PBS was added per well; cells were lysed after $4 \mathrm{~h}$ by addition of $200 \mu \mathrm{l}$ dimethyl sulfoxide (DMSO; Sigma). Absorbance was measured at $570 \mathrm{~nm}$.

Colony formation assay. After transfection, the cells were seeded in 6-well plates at a density of 3,000 cells/well and cultured for 9-14 days until visible colonies appeared. Then the cells were stained with crystal violet. The number of colonies was counted only if they contained more than 50 cells.

Cell cycle assays. Cell cycle distribution was detected by flow cytometry (FCM). After 48 h of transient transfections, the cells were harvested by trypsinisation and fixed in $70 \%$ ice-cold ethanol the overnight. Then cells were washed with cold PBS and resuspended in propidium iodide (PI) nuclear staining for cell cycle analysis.

Western blotting. Whole cell extracts were prepared in RIPA buffer containing protease inhibitor PMSF (both from Beyotime, Shanghai, China). Protein concentration was measured using the BCA assay (Beyotime) according to the manufacturer's instructions. Total protein was electrophoresed by SDS-PAGE. Then the proteins were transferred to a polyvinylidene fluoride membrane (Millipore, Billerica, MA, USA) and blocked for $1 \mathrm{~h}$ with $5 \%$ skim milk at room temperature. Incubation with primary antibodies was conducted overnight at $4^{\circ} \mathrm{C}$. The blots were incubated with horseradish peroxidase labelled secondary antibodies and the signal was detected using ECL (Beyotime). The following antibodies were used for western blotting: rabbit anti-GAPDH (1:500; Xianzhi Biotechnology, Hangzhou, China), rabbit anti-HMGA1 (1:500; Cell Signaling Technology, USA), HRP-labelled goat anti-rabbit secondary antibody (1:3,000; Zhongshan Golden Bridge Biotechnology, Beijing, China).

Construction of the plasmid vectors. HMGA1 3'UTR-luciferase reporter vectors were cloned into psiCHECK-2 ${ }^{\mathrm{TM}}$ vectors. In brief, wide and mutant 3'UTR regions were chemically synthesised and cloned into the XhoI and NotI sites of the psiCHECK-2 ${ }^{\text {TM }}$ vector (Promega, Madison, WI, USA).

Luciferase assay. For the luciferase reporter assay, the cells were cultured in 24-well plates and transiently co-transfected with $50 \mathrm{nM}$ miRNA (miR-195 mimics or scrambled miR-195 negative control) and $250 \mathrm{ng}$ reporter vectors (wild-type reporter vectors or mutant-type reporter vectors), using Lipofectamine $^{\mathrm{TM}}$ 2000. Luciferase activities were measured using a Dual-Luciferase assay kit (Promega) according to the manufacturer's instructions at $48 \mathrm{~h}$ post-transfection

Lentiviral packaging and establishment of stable cell lines. The lentiviral packaging kit was purchased from Open Biosystems (Huntsville, AL, USA). The lentivirus carrying hsa-miR-195 or hsa-miR-negative control (NC) was packaged following the manufacturer's manual. Stable cell lines were established by infecting the lentivirus into PC-3 cells and selection by puromycin.

Tumor growth assay in mice and immunohistochemical staining. Nude mice (female BALB/c-nu, 4-weeks old) were purchased from the Shanghai Experimental Animal Center (Chinese Academy of Sciences, Shanghai, China), and maintained under special pathogen-free (SPF) conditions. Six mice were randomly divided into two groups. PC-3 cells stably expressing miR-195 were injected subcutaneously into the flank of nude mice $\left(4 \times 10^{6}\right.$ cells in $\left.100 \mu \mathrm{l}\right)$. PC-3 cells stably expressing miR-NC were used as the negative control. Tumor size was measured using a vernier caliper every week, and tumor volume was calculated according to the formula: Volume $=0.5 \mathrm{x}$ length $\mathrm{x}$ width ${ }^{2}$. Immunohistochemical staining assay was performed as previously described (2).

\section{Results}

High level of miR-195 is associated with a good prognosis of $P C a$. In the GSE21304 data, we analyzed the miR-195 expression pattern in different pathological grade $\mathrm{PCa}$ tissues and found that miR-195 expression was downregulated in high grade PCa tissues compared to low grade tissues (Fig. 1A). Next, the relationship between miR-195 expression and patient prognosis was investigated. The result indicated that a high level of miR-195 was associated with longer disease-free survival (Fig. 1B).

miR-195 overexpression inhibits proliferation and induces cell cycle arrest in prostate cancer cells. To assess the role 

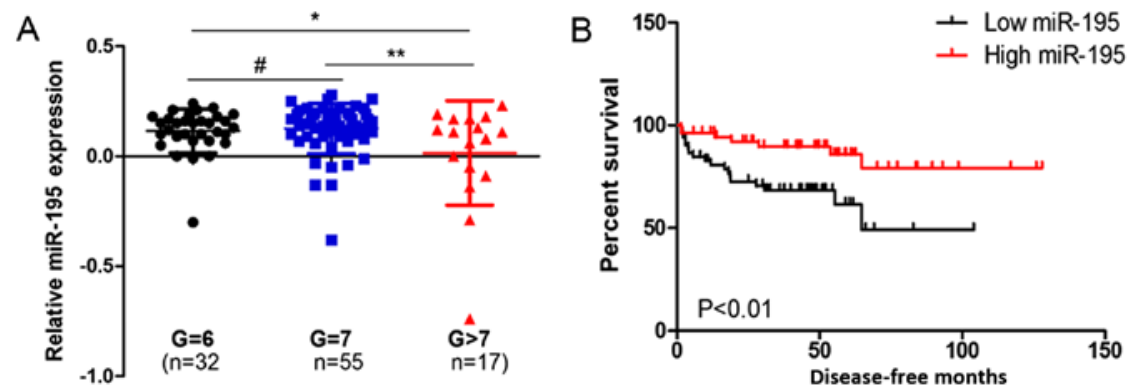

Figure 1. miR-195 expression is associated with the prognosis of PCa patients. (A) miR-195 expression was found to be downregulated in high grade PCa tissues compared to low grade tissues. (B) A high level of miR-195 was associated with longer disease-free survival.

A

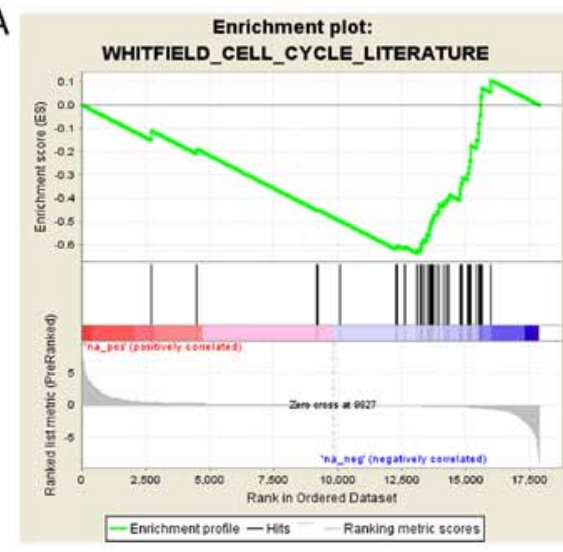

B
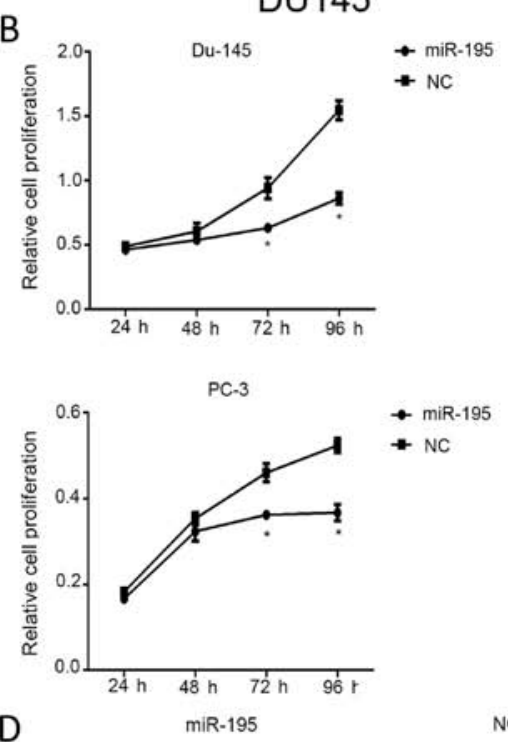

D
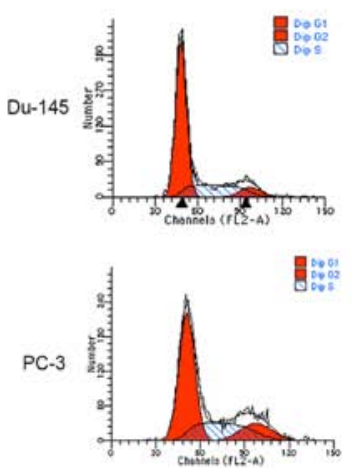

C

NC

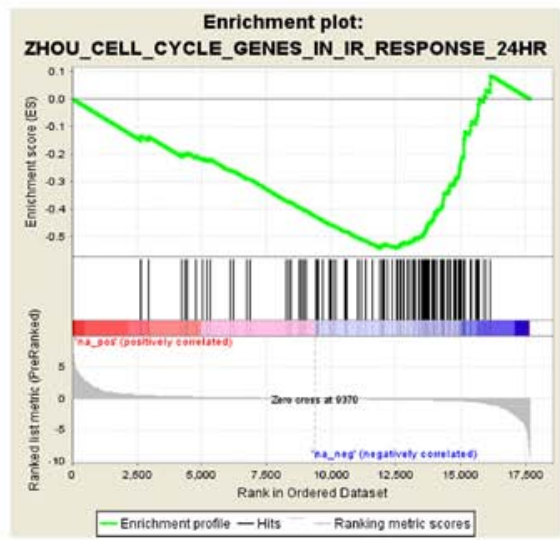

PC-3
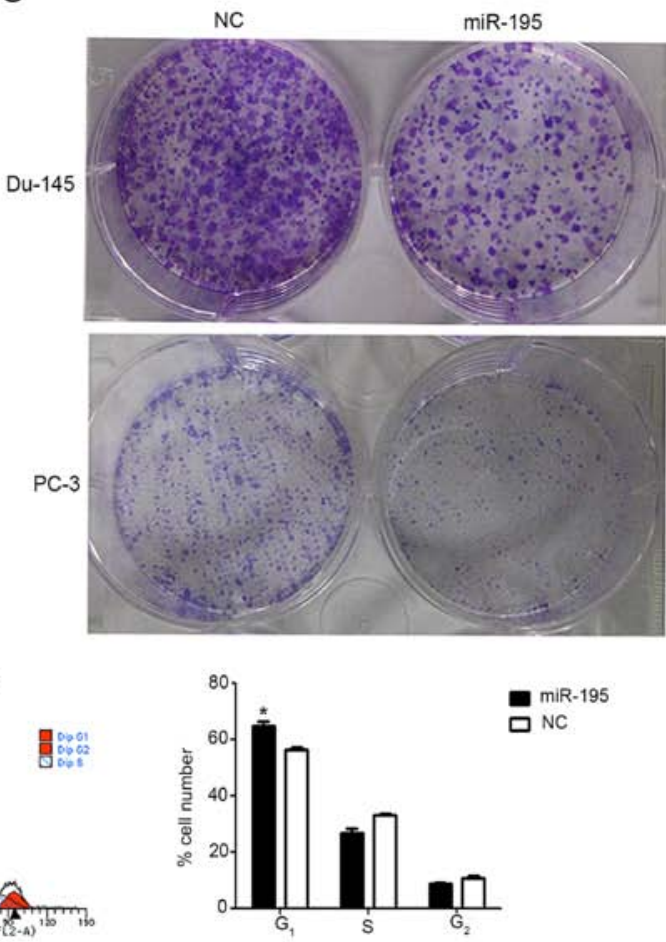

miR-195
nC

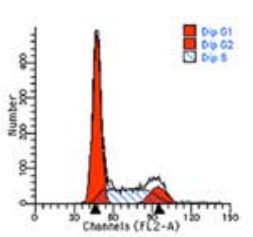

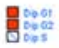

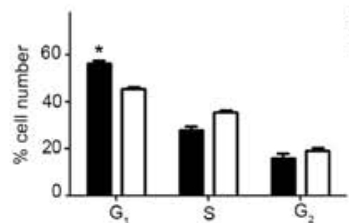

Figure 2. miR-195 is associated with the cell cycle. (A) GSEA analysis showed that there was negatively enriched expression of gene sets involved in cell cycle progression in the miR-195-transfected cells. (B and C) Cell growth ability was determined by MTT assay and colony formation assay. (D) Cells were transfected with the miR-195 mimics, and the distribution of cells within the stages of the cell cycle was determined. 

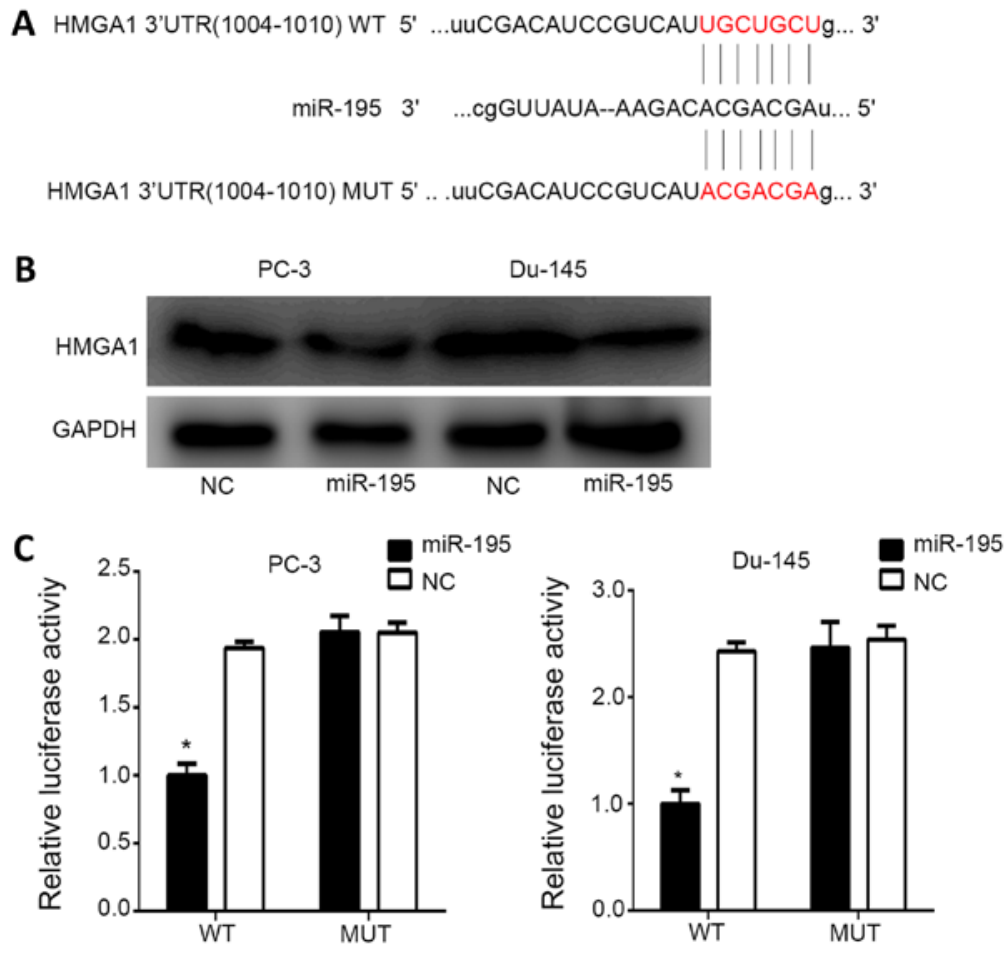

Figure 3. miR-195 directly targets HMGA1. (A) Putative binding sites of miR-195 within the HMGA1 3'UTR, as predicted by TargetScan Human and miRanda. (B) HMGA1 protein level was measured in PCa cells at $48 \mathrm{~h}$ post-transfection. (C) Luciferase reporter assays were performed to detect the regulatory mechanism between miR-195 and its binding site within the HMGA1 mRNA 3'UTR.

of miR-195, we overexpressed miR-195 in prostate cancer cell lines (DU145 and PC3) followed by RNA sequence and bioinformational analysis. GSEA analysis showed that there was negatively enriched expression of gene sets involved in cell cycle progression in the miR-195-transfected cells (Fig. 2A). Consistent with the GSEA analysis, miR-195 overexpression inhibited DU145 and PC3 cell proliferation in the MTT assay (Fig. 2B). miR-195 overexpression also decreased clonogenicity of the DU145 and PC3 cells compared to that noted in the miR-NC (Fig. 2C). FCM analysis showed that overexpression of miR-195 led to a significant increase in the percentage of G0/G1 phase DU145 and PC3 cells (Fig. 2D). This indicated that miR-195 overexpression suppressed the growth of prostate cancer cells.

miR-195 targets HMGAl in prostate cancer cells. To find the target of miR-195, bioinformatic analysis was performed using TargetScan Human 6.2 and miRanda. HMGA1 was found to possess potential miR-195 target sites within its 3'UTR (Fig. 3A). Guided by this finding, we performed western blot analysis in the DU145 and PC3 cells that were transfected with miR-195 or miR-NC. The results showed that overexpression of miR-195 inhibited HMGA1 expression (Fig. 3B). In order to investigate HMGA1 as a direct target of miR-195, transient transfection of the HMGA1 3'UTR plasmid along with miR-195/miR-NC was performed in the DU145 and PC3 cells. A significant decrease was observed in luciferase activities when compared with the mutant plasmid (Fig. 3C). These results showed that HMGA1 is a direct target of miR-195.

The role of HMGAl in PCa. In the GSE35988 dataset, we initially analyzed the HMGA1 expression pattern in benign prostate hyperplasia (BPH) tissues and different PCa tissues and found that HMGA1 expression was elevated in the CRPC tissues compared to the level noted in the BPH tissues. Furthermore, HMGA1 expression was significantly increased in the CRPC tissues when compared with that noted in the androgen-dependent prostate cancer (ADPC) tissues (Fig. 4A). GSEA was used to evaluate the pathways that were differentially expressed between patients with high levels of HMGA1 expression and those with low levels of HMGA1 expression. The data revealed that HMGA1 regulates genes primarily associated with PCa cell cycle progression (Fig. 4B). Next, we investigated the correlation between HMGA1 expression and survival using Kaplan-Meier survival curve analysis with a log-rank comparison. PCa samples expressing higher than median levels of HMGA1 were associated with decreased biochemical relapse-free survival and overall survival relative to those with HMGA1 levels lower than the median $(\mathrm{P}<0.05)$ in the Oncomine data (Fig. 4C and D).

Then, HMGA1 was knocked down in the DU145 and PC3 cells by introducing siRNA. The knockdown efficiency was verified through western blot analysis and reduced expression of HMGA1 was observed (Fig. 5B). MTT assay showed that low expression of HMGA1 inhibited the proliferation of the PCa cells (Fig. 5A). FCM analysis also indicated that low expression of HMGA1 induced G0/G1 phase arrest (Fig. 5C).

MiR-195 inhibits PCa tumorigenesis and downregulates HMGA1 in vivo. In the previous experiment, miR-195 was found to be downregulated in human PCa tissues and to play an important role in proliferation and cell cycle distribution. We further examined the effect of miR-195 in a nude mouse PCa xenograft model. Overexpression of miR-195 inhibited 

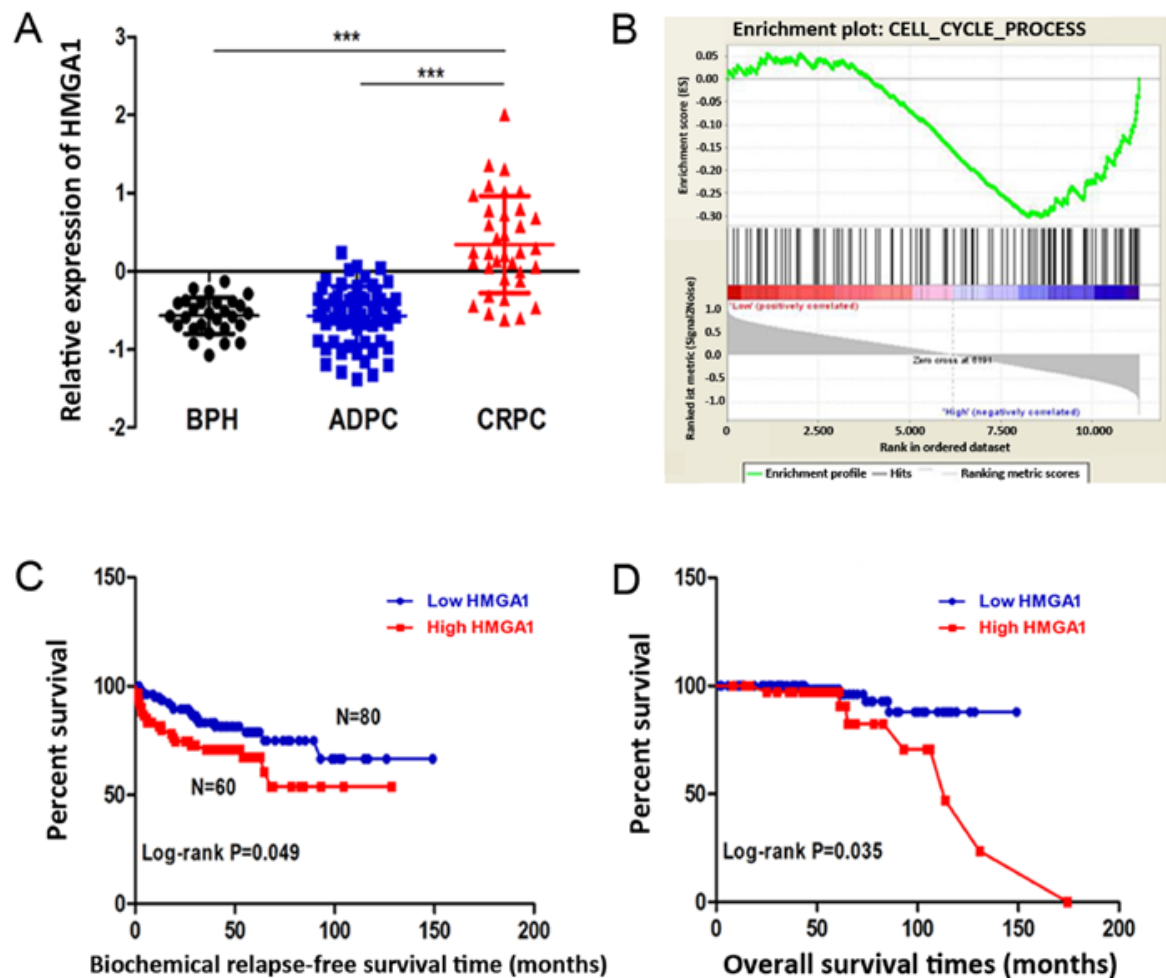

Figure 4. Increased HMGA1 expression confers poor prognosis in PCa patients. (A) The levels of HMGA1 were analyzed in PCa and BPH tissues of the GSE dataset. (B) GSEA analysis showed that there was enriched expression of gene sets involved in PCa cell cycle progression in high-HMGA1 expression samples. (C and D) Kaplan-Meier survival curves for HMGA1 expression in PCa tissues of the Oncomine dataset. BPH, benign prostate hyperplasia; ADPC, androgen-dependent prostate cancer; CRPC, castration-resistant prostate cancer.
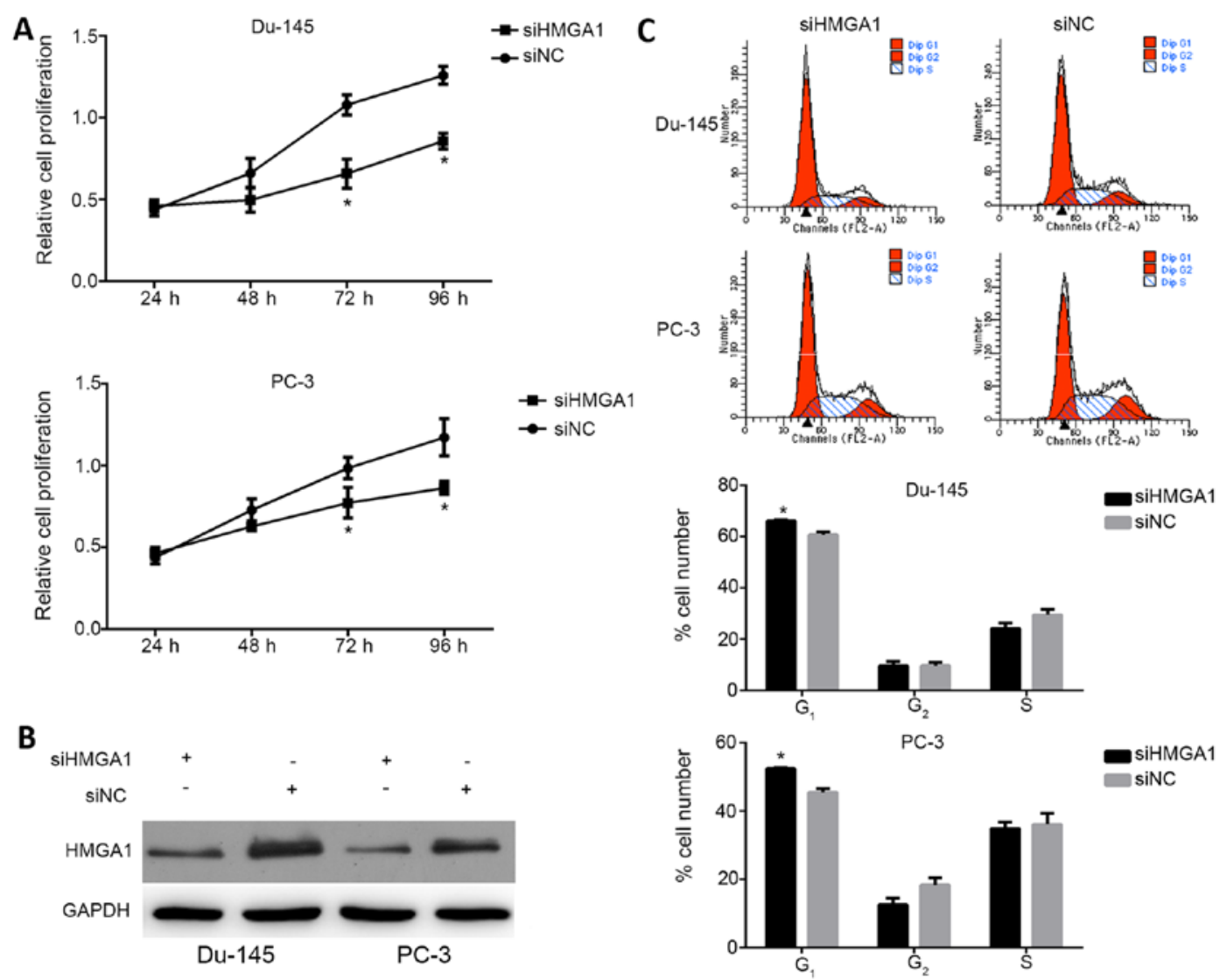

Figure 5. HMGA1-siRNA inhibits PCa progression. (A) Cell proliferation was determined by MTT assay. (B) Western blot analyses were used to determine HMGA1 expression changes following cell transfection with siHMGA1. (C) Cells were transfected with siHMGA1, and the distribution of cells within the stages of the cell cycle was determined. 


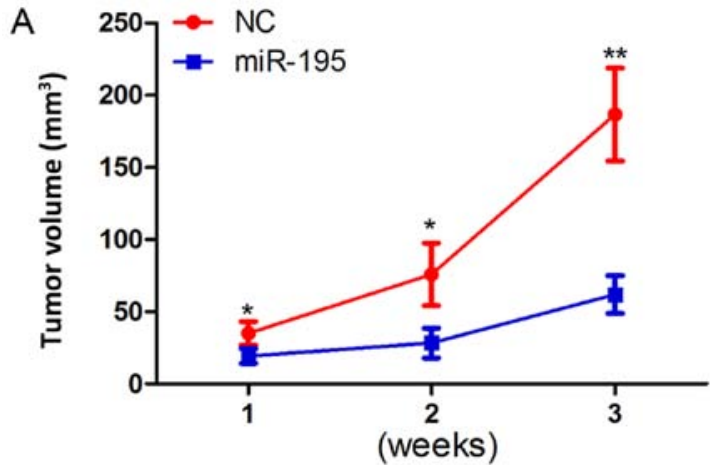

B

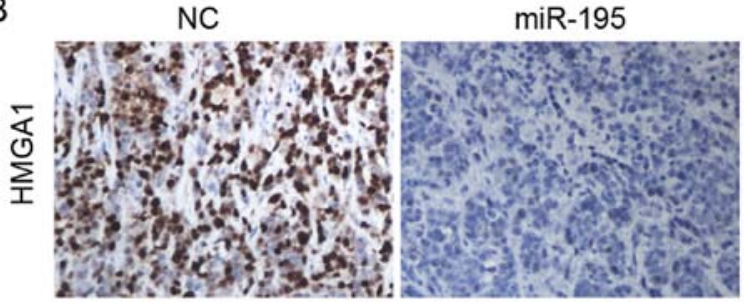

Figure 6. miR-195/HMGA1 axis was confirmed in nude mouse tumor xenograft. (A) Nude mice were injected subcutaneously with PC-3 cells stably expressing miR-195 (miR-195) or miR-negative control (NC). The width and length of tumors were measured every week. ${ }^{*} \mathrm{P}<0.05$, significant difference when compared to the NC group. (B) The expression level of HMGA1 was analyzed in tumor tissues by immunohistochemistry.

PCa xenograft growth in vivo (Fig. 6A). Immunohistochemical analysis revealed that HMGA1 was markedly decreased following miR-195 treatment (Fig. 6B).

\section{Discussion}

miR-195, derived from the miR-497/miR-195 locus at human chromosome $17 \mathrm{p} 13.1$, has been found to be aberrantly deregulated in tumorigenesis (11). For example, it is upregulated in metastatic melanoma (10) and some cases of lung cancer (16), and hepatocellular carcinoma (17). By contrast, it is preferentially downregulated in breast cancer (18), gastric cancer $(17,19)$, colorectal cancer (20-22), and bladder cancer $(23,24)$. However, inconsistent and inconclusive results have been reported concerning the expression and function of miR-195 in PCa $(17,25)$. These controversial observations indicate the complexity of miR-195 during tumorigenesis and development and also call for further investigation of the role in PCa. In the present study, we found that miR-195 expression levels were decreased in human PCa samples and were positively correlated with prognosis. Overexpression of miR-195 significantly suppressed the cell cycle and proliferation of PCa cells. In addition, our results in PCa cells indicated that miR-195 targets HMGA1 and negatively regulates its expression at the translational level which indicates that miR-195 may suppress PCa malignant progression by downregulating the HMGA1 oncogene.

HMGA proteins are encoded by two genes, HMGA1 and HMGA2, located at chromosome 6p21 and 12q13-15, respectively. Numerous studies have confirmed the association of HMGA, in particular HMGA1 overexpression, with a high malignant phenotype as outlined by chemoresistance, the spread of metastases, and a global poor survival (26). For example, overexpression of HMGA1 in colon carcinoma was found to be strongly associated with invasive ability, staining being more intense in invasion-positive cases in comparison to invasion-negative ones (27), in advanced stage (T3 and T4 tumors) and with the presence of distant, but not regional, metastases. Several reports have indicated that HMGA1 is abundantly expressed in pancreas adenocarcinomas, where overexpression of HMGA1 correlates with advanced grade and, though less frequently, in pancreas intraepithelial neoplasias (28). Moreover, HMGA1 is not expressed in the normal epithelium surface where adenocarcinomas originate, but it was found to be highly expressed in invasive ovarian carcinomas, and weakly expressed in ovarian carcinomas with low invasive potential (29). To evaluate the expression and role of HMGA1 in PCa, we downloaded mRNA expression and clinical follow-up data from the Oncomine and GSE database. Our analysis showed upregulation of HMGA1 in CRPC samples in comparison to ADPC and BPH tissues. In addition, high HMGA1 expression was associated with the poor prognosis of PCa patients. Moreover, HMGA1 knockdown significantly decreased the potential of cell proliferation and cell cycle progression in the PCa cells in vitro, which had effects similar to those for miR-195 overexpression.

In conclusion, the results presented here demonstrate that miR-195 has significant biological effects on PCa development. Overexpression of miR-195 downregulated the expression of HMGA1 protein, suggesting that miR-195 functions as a tumor suppressor probably through downregulation of HMGA1 in PCa. Furthermore, there are other putative miR-195 target genes which could potentially be key players in the malignant progression of PCa cells. miR-195 may prove to be a promising gene therapeutic agent.

\section{Acknowledgements}

This study was supported by the National Natural Science Foundation of China (81370849, 81300472, and 81572517), the Natural Science Foundation of Jiangsu Province (BL2013032 and BK2012336) and Nanjing City (201201053) and Southeast University (3290002402), Science Foundation of Ministry of Education of China (20120092120071).

\section{References}

1. Siegel R, Ma J, Zou Z and Jemal A: Cancer statistics, 2014. CA Cancer J Clin 64: 9-29, 2014.

2. Tao T, Liu D, Liu C, Xu B, Chen S, Yin Y, Ang L, Huang Y, Zhang $X$ and Chen $M$ : Autoregulatory feedback loop of $\mathrm{EZH} 2 / \mathrm{miR}-200 \mathrm{c} / \mathrm{E} 2 \mathrm{~F} 3$ as a driving force for prostate cancer development. Biochim Biophys Acta 1839: 858-865, 2014.

3. Zhu C, Li J, Ding Q, Cheng G, Zhou H, Tao L, Cai H, Li P, Cao Q, Ju X, et al: miR-152 controls migration and invasive potential by targeting TGF $\alpha$ in prostate cancer cell lines. Prostate 73: 1082-1089, 2013.

4. Liu D, Tao T, Xu B, Chen S, Liu C, Zhang L, Lu K, Huang Y, Jiang L, Zhang X, et al: miR-361-5p acts as a tumor suppressor in prostate cancer by targeting signal transducer and activator of transcription-6 (STAT6). Biochem Biophys Res Commun 445: 151-156, 2014.

5. Xu B, Wang N, Wang X, Tong N, Shao N, Tao J, Li P, Niu X, Feng $\mathrm{N}$, Zhang L, et al: miR-146a suppresses tumor growth and progression by targeting EGFR pathway and in a p-ERK-dependent manner in castration-resistant prostate cancer. Prostate 72: 1171-1178, 2012. 
6. Tao T, Li G, Dong Q, Liu D, Liu C, Han D, Huang Y, Chen S, Xu B and Chen M: Loss of SNAIL inhibits cellular growth and metabolism through the miR-128-mediated RPS6KB1/HIF-1 $\alpha / \mathrm{PKM} 2$ signaling pathway in prostate cancer cells. Tumour Biol 35: 8543-8550, 2014.

7. Griffiths-Jones S, Saini HK, van Dongen S and Enright AJ: miRBase: Tools for microRNA genomics. Nucleic Acids Res 36 (Database): D154-D158, 2008.

8. Finnerty JR, Wang WX, Hébert SS, Wilfred BR, Mao G and Nelson PT: The miR-15/107 group of microRNA genes: Evolutionary biology, cellular functions, and roles in human diseases. J Mol Biol 402: 491-509, 2010.

9. Zanette DL, Rivadavia F, Molfetta GA, Barbuzano FG, Proto-Siqueira R, Silva-Jr WA, Falcão RP and Zago MA miRNA expression profiles in chronic lymphocytic and acute lymphocytic leukemia. Braz J Med Biol Res 40: 1435-1440, 2007.

10. Bhattacharya A, Schmitz U, Wolkenhauer O, Schönherr M, Raatz Y and Kunz M: Regulation of cell cycle checkpoint kinase WEE1 by miR-195 in malignant melanoma. Oncogene 32 : 3175-3183, 2013.

11. Wang X, Wang J, Ma H, Zhang J and Zhou X: Downregulation of miR-195 correlates with lymph node metastasis and poor prognosis in colorectal cancer. Med Oncol 29: 919-927, 2012.

12. Xu T, Zhu Y, Xiong Y, Ge YY, Yun JP and Zhuang SM: MicroRNA-195 suppresses tumorigenicity and regulates G1/S transition of human hepatocellular carcinoma cells. Hepatology 50: 113-121, 2009.

13. Soon PS, Tacon LJ, Gill AJ, Bambach CP, Sywak MS, Campbell PR, Yeh MW, Wong SG, Clifton-Bligh RJ, Robinson BG, et al: miR-195 and miR-483-5p identified as predictors of poor prognosis in adrenocortical cancer. Clin Cancer Res 15: 7684-7692, 2009.

14. Grasso CS, Wu YM, Robinson DR, Cao X, Dhanasekaran SM, Khan AP, Quist MJ, Jing X, Lonigro RJ, Brenner JC, et al: The mutational landscape of lethal castration-resistant prostate cancer. Nature 487: 239-243, 2012.

15. Taylor BS, Schultz N, Hieronymus H, Gopalan A, Xiao Y, Carver BS, Arora VK, Kaushik P, Cerami E, Reva B, et al: Integrative genomic profiling of human prostate cancer. Cancer Cell 18: 11-22, 2010.

16. Volinia S, Calin GA, Liu CG, Ambs S, Cimmino A, Petrocca F, Visone R, Iorio M, Roldo C, Ferracin M, et al: A microRNA expression signature of human solid tumors defines cancer gene targets. Proc Natl Acad Sci USA 103: 2257-2261, 2006.

17. Ding J, Huang S, Wang Y, Tian Q, Zha R, Shi H, Wang Q, Ge C, Chen T, Zhao Y, et al: Genome-wide screening reveals that miR-195 targets the TNF- $\alpha / N F-\kappa B$ pathway by down-regulating $\mathrm{I} \kappa \mathrm{B}$ kinase alpha and TAB3 in hepatocellular carcinoma. Hepatology 58: 654-666, 2013.

18. Li D, Zhao Y, Liu C, Chen X, Qi Y, Jiang Y, Zou C, Zhang X, Liu S, Wang X, et al: Analysis of miR-195 and miR-497 expression, regulation and role in breast cancer. Clin Cancer Res 17: 1722-1730, 2011.
19. Deng H, Guo Y, Song H, Xiao B, Sun W, Liu Z, Yu X, Xia T, Cui L and Guo J: MicroRNA-195 and microRNA-378 mediate tumor growth suppression by epigenetical regulation in gastric cancer. Gene 518: 351-359, 2013.

20. Chen X, Guo X, Zhang H, Xiang Y, Chen J, Yin Y, Cai X, Wang K, Wang G, Ba Y, et al: Role of miR-143 targeting KRAS in colorectal tumorigenesis. Oncogene 28: 1385-1392, 2009.

21. Guo ST, Jiang CC, Wang GP, Li YP, Wang CY, Guo XY Yang RH, Feng Y, Wang FH, Tseng HY, et al: MicroRNA-497 targets insulin-like growth factor 1 receptor and has a tumour suppressive role in human colorectal cancer. Oncogene 32: 1910-1920, 2013.

22. Liu L, Chen L, Xu Y, Li R and Du X: microRNA-195 promotes apoptosis and suppresses tumorigenicity of human colorectal cancer cells. Biochem Biophys Res Commun 400: 236-240, 2010

23. Lin Y, Wu J, Chen H, Mao Y, Liu Y, Mao Q, Yang K, Zheng X and Xie L: Cyclin-dependent kinase 4 is a novel target in micoRNA-195-mediated cell cycle arrest in bladder cancer cells. FEBS Lett 586: 442-447, 2012

24. Itesako T, Seki N, Yoshino H, Chiyomaru T, Yamasaki T, Hidaka H, Yonezawa T, Nohata N, Kinoshita T, Nakagawa M, et al: The microRNA expression signature of bladder cancer by deep sequencing: The functional significance of the miR-195/497 cluster. PLoS One 9: e84311, 2014.

25. Porkka KP, Pfeiffer MJ, Waltering KK, Vessella RL, Tammela TL and Visakorpi T: MicroRNA expression profiling in prostate cancer. Cancer Res 67: 6130-6135, 2007.

26. Katoh M: Cardio-miRNAs and onco-miRNAs: Circulating miRNA-based diagnostics for non-cancerous and cancerous diseases. Front Cell Dev Biol 2: 61, 2014.

27. Zhang L, Kim M, Choi YH, Goemans B, Yeung C, Hu Z, Zhan S, Seth P and Helman LJ: Diminished G1 checkpoint after gamma-irradiation and altered cell cycle regulation by insulin-like growth factor II overexpression. J Biol Chem 274 13118-13126, 1999.

28. Piscuoglio S, Zlobec I, Pallante P, Sepe R, Esposito F, Zimmermann A, Diamantis I, Terracciano L, Fusco A and Karamitopoulou E: HMGA1 and HMGA2 protein expression correlates with advanced tumour grade and lymph node metastasis in pancreatic adenocarcinoma. Histopathology 60: 397-404, 2012

29. Masciullo V, Baldassarre G, Pentimalli F, Berlingieri MT, Boccia A, Chiappetta G, Palazzo J, Manfioletti G, Giancotti V, Viglietto G, et al: HMGA1 protein over-expression is a frequent feature of epithelial ovarian carcinomas. Carcinogenesis 24 : 1191-1198, 2003. 